MATHEMATICS OF COMPUTATION

Volume 80, Number 274, April 2011, Pages 973-994

S $0025-5718(2010) 02380-4$

Article electronically published on October 18, 2010

\title{
SUBSEQUENCE CONVERGENCE IN SUBDIVISION
}

\author{
DETER DE WET
}

\begin{abstract}
We study the phenomenon that regularly spaced subsequences of the control points in subdivision may converge to scalar multiples of the same limit function, even though subdivision itself is divergent. We present different sets of easily checkable sufficient conditions for this phenomenon (which we term subsequence convergence) to occur, study the basic properties of subsequence convergence, show how certain results from subdivision carry over to this case, show an application for decorative effects, and use our results to build nested sets of refinement masks, which provide some insight into the structure of the set of refinable functions. All our results are formulated for a general integer dilation factor.
\end{abstract}

\section{INTRODUCTION}

In this paper we consider univariate subdivision schemes with a general integer dilation factor, i.e. for an integer $p \in \mathbb{Z}, p \geq 2$, called the dilation factor, and a bi-infinite complex-valued sequence $a=\left(a_{j}: j \in \mathbb{Z}\right)$, called the (subdivision) mask, we define the subdivision operator $S_{a, p}$ by

$$
\left(S_{a, p} c\right)_{j}=\sum_{i} a_{j-p i} c_{i}, \quad j \in \mathbb{Z}, \quad c \in M(\mathbb{Z}),
$$

where, as throughout this paper, $M(\mathbb{Z})$ denotes the set of complex-valued bi-infinite sequences and $\sum_{j}$ denotes $\sum_{j \in \mathbb{Z}}$. Subdivision plays an important role in the construction of curves and surfaces in CAGD (see e.g. [9]). We shall only consider masks in $M_{0}(\mathbb{Z})$, which denotes the set of sequences $a \in M(\mathbb{Z})$ for which $\operatorname{supp}(a)=\left\{j \in \mathbb{Z}: a_{j} \neq 0\right\}$, called the support of $a$, is finite.

For a given initial sequence $c \in M(\mathbb{Z})$, we recursively define

$$
c^{(0)}=c ; \quad c^{(r)}=S_{a, p} c^{(r-1)}, r \in \mathbb{N} .
$$

We call this process the subdivision scheme $\left(S_{a, p}, c\right)$.

We say that the subdivision scheme $\left(S_{a, p}, c\right)$ converges if there exists a nonzero function $\Phi \in C(\mathbb{R})$, called the limit function of the subdivision scheme, such that

$$
\sup _{j \in \mathbb{Z}}\left|\Phi\left(\frac{j}{p^{r}}\right)-c_{j}^{(r)}\right| \rightarrow 0, \quad r \rightarrow \infty .
$$

The following necessary condition for subdivision convergence is known (see [3, Proposition 2.1] for the case $p=2$ and [7, Proposition 1] for the extension to $p \in \mathbb{Z}$ and the multivariate case).

Received by the editor April 16, 2008 and, in revised form, April 29, 2009.

2010 Mathematics Subject Classification. Primary 65D10, 65D17, 41A99.

(C)2010 American Mathematical Society 
Theorem 1.1. For $p \in \mathbb{Z}, p \geq 2$, and $a \in M_{0}(\mathbb{Z})$, suppose there exists a sequence $c \in l^{\infty}(\mathbb{Z})$ such that the subdivision scheme $\left(S_{a, p}, c\right)$ converges to $\Phi$. Then the sum rules

$$
\sum_{j} a_{p j+l}=1, \quad l \in\{0, \ldots, p-1\}
$$

hold for the mask a.

During numerical experiments with masks not satisfying the requirements of Theorem 1.1. it was detected that sometimes the subdivision algorithm "converges" to two or more limits. To make the meaning of this clear, consider the following example, which is a modification of [10, Example 2.2].

Example 1.2. Let $p=2$ and let the mask $a$ be given by

$$
a_{0}=a_{4}=\frac{3}{4}, \quad a_{1}=a_{5}=-\frac{1}{4}, \quad a_{2}=\frac{3}{2}, \quad a_{3}=-\frac{1}{2} .
$$

Observe that $\sum_{j} a_{2 j} \neq 1$ and $\sum_{j} a_{2 j+1} \neq 1$. We take the initial sequence $c=\delta$, with $\delta$ denoting here and elsewhere the Kronecker delta sequence, i.e.

$$
\delta_{0}=1 \text { and } \delta_{j}=0, j \neq 0 \text {. }
$$

As can be seen from Figure 1.1(a), which shows the fourth iteration of subdivision, the subdivision algorithm is divergent and appears to oscillate between two functions on consecutive control points. In Figure 1.1(b), we show separate plots connecting the odd-indexed points and even-indexed points, respectively, of the eighth iteration of subdivision. We see that the two functions thus obtained seem to be scalar multiples of one another, with the one built from the even indices being minus three times the other one.

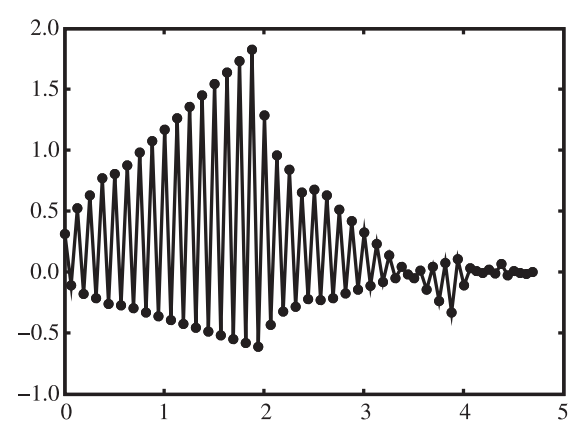

(a)

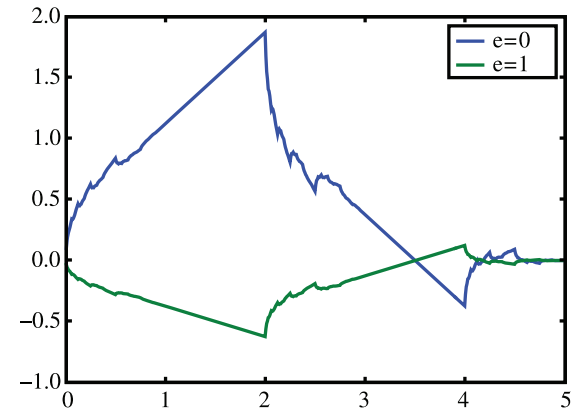

(b)

Figure 1.1. Plots of (a) $S_{a}^{4} \delta$ and (b) $\left(S_{a}^{8} \delta\right)_{2+e}, e=0,1$, in Example 1.2 .

In this paper, we study this phenomenon that regularly spaced subsequences of the control points in subdivision may converge to scalar multiples of the same limit function. This we term subsequence convergence, of which we will give a formal definition after establishing further necessary notation and preliminary results. 
Further notation. Throughout this work, $\chi$ shall denote the characteristic function of $[0,1)$.

We write $\sup _{j}$ for $\sup _{j \in \mathbb{Z}}$. We let $\mathbb{Z}_{+}=\mathbb{N} \cup\{0\}$ denote the set of nonnegative integers. For $x \in \mathbb{R},\lfloor x\rfloor$ denotes the largest integer $\leq x$ and $\lceil x\rceil$ denotes the smallest integer $\geq x$.

For $m \in \mathbb{N}, \mathbb{Z}_{m}$ denotes the set $\{0, \ldots, m-1\}$. We shall use the facts that

$$
\mathbb{Z}_{m n}=\left\{j m+l: j \in \mathbb{Z}_{n}, l \in \mathbb{Z}_{m}\right\} \quad \text { and } \quad \mathbb{Z}=\left\{j m+l: j \in \mathbb{Z}, l \in \mathbb{Z}_{m}\right\},
$$

which allows us to partition sums into appropriate double summations and vice versa.

For $j, m \in \mathbb{Z}, j \bmod m$ denotes the remainder in $\mathbb{Z}_{m}$ when $j$ is divided by $m$, that is

$$
j \bmod m=j-m\left\lfloor\frac{j}{m}\right\rfloor, \quad j, m \in \mathbb{Z} .
$$

We let the set of functions from $\mathbb{R}$ to $\mathbb{C}$ be denoted by $M(\mathbb{R})$. The support of a function $f$ is the set $\overline{\{x \in \mathbb{R}: f(x) \neq 0\}}$ and is denoted by $\operatorname{supp}(f)$. The set of functions in $M(\mathbb{R})$ with compact support is denoted by $M_{0}(\mathbb{R})$, while $M_{+}(\mathbb{R})$ denotes the set of functions in $M(\mathbb{R})$ that vanish left of the origin. We set $M_{0}^{+}(\mathbb{R})=$ $M_{0}(\mathbb{R}) \bigcap M_{+}(\mathbb{R})$.

For nonzero $a \in M_{0}(\mathbb{Z})$, we define $\downarrow a \downarrow=\min \left\{j \in \mathbb{Z}: a_{j} \neq 0\right\}$ and $\uparrow a \uparrow=$ $\max \left\{j \in \mathbb{Z}: a_{j} \neq 0\right\}$. We let $M_{0}^{+}(\mathbb{Z})$ denote the set $\left\{a \in M_{0}(\mathbb{Z}) \backslash\{0\}: \downarrow a \downarrow=0\right\}$. Let $\Delta$ denote the backward difference operator defined by $(\Delta c)_{j}=c_{j}-c_{j-1}, j \in \mathbb{Z}$, $c \in M(\mathbb{Z})$, and by $\Delta^{\infty}(\mathbb{Z})$ we mean the subspace $\left\{c \in M(\mathbb{Z}): \Delta c \in l^{\infty}(\mathbb{Z})\right\}$ of $M(\mathbb{Z})$.

For $a \in M(\mathbb{Z})$ and $k \in \mathbb{C}, k a$ denotes the sequence defined by $(k a)_{j}=k a_{j}, j \in \mathbb{Z}$.

We shall sometimes exploit the one-to-one correspondence between Laurent polynomials and compactly supported sequences in our proofs. To this end, we define the following operators.

Definition 1.3. For a sequence $p \in M_{0}(\mathbb{Z})$, define the Laurent polynomial $\tilde{p}$ by

$$
\widetilde{p}(z)=\sum_{j} p_{j} z^{j}, \quad z \in \mathbb{C} \backslash\{0\} .
$$

Definition 1.4. For a Laurent polynomial $P$, let $[P] \in M_{0}(\mathbb{Z})$ denote the coefficient sequence of $P$, i.e. for $j \in \mathbb{Z},[P]_{j}$ equals the coefficient of $z^{j}$ in $P$.

Observe that $\bar{p}$ is actually a polynomial if $p \in M_{0}^{+}(\mathbb{Z})$, while, for nonzero Laurent polynomials $P$ and $Q$,

$$
\uparrow[P Q] \uparrow=\uparrow[P] \uparrow+\uparrow[Q] \uparrow \quad \text { and } \quad \downarrow[P Q] \downarrow=\downarrow[P] \downarrow+\downarrow[Q] \downarrow .
$$

We will also make frequent use of the following operator and its properties.

Definition 1.5. For a Laurent polynomial $P$ and $m \in \mathbb{N}$, define $P^{\langle m\rangle}$ to be the Laurent polynomial given by

$$
P^{\langle m\rangle}(z)=P\left(z^{m}\right), \quad z \in \mathbb{C} \backslash\{0\} .
$$

Note that $P^{\langle 1\rangle}=P$. Also, provided $P \neq 0$, we have

$$
\uparrow\left[P^{\langle m\rangle}\right] \uparrow=m \uparrow[P] \uparrow \quad \text { and } \quad \downarrow\left[P^{\langle m\rangle}\right] \downarrow=m \downarrow[P] \downarrow .
$$

The next lemma states some further properties of this operator, which can easily be verified. 
Lemma 1.6. Suppose $P$ and $Q$ are Laurent polynomials and $m, n \in \mathbb{N}$. Then the following identities hold:

$$
\begin{aligned}
\left(P^{\langle m\rangle}\right)^{\langle n\rangle} & =\left(P^{\langle n\rangle}\right)^{\langle m\rangle}=P^{\langle m n\rangle} ; \\
(P Q)^{\langle m\rangle} & =P^{\langle m\rangle} Q^{\langle m\rangle} ; \\
{\left[P Q^{\langle m\rangle}\right]_{j} } & =\sum_{k}[P]_{j-k m}[Q]_{k}, \quad j \in \mathbb{Z} .
\end{aligned}
$$

Elementary polynomials. The following class of polynomials will prove useful in various proofs. Define, for a given $m \in \mathbb{Z}_{+}$, the polynomial $E_{m}$ by

$$
E_{m}(z)=\frac{1}{m+1} \sum_{j=0}^{m} z^{j}=\frac{1}{m+1} \prod_{j=1}^{m}\left(z-e^{2 j \pi i /(m+1)}\right), \quad z \in \mathbb{C},
$$

where the second equality follows from the fact that the first equality yields

$$
E_{m}(z)=\frac{1-z^{m+1}}{(m+1)(1-z)}, \quad z \in \mathbb{C} \backslash\{1\}, \quad m \in \mathbb{Z}_{+} .
$$

Note that $E_{m}(1)=1, m \in \mathbb{N}$, and that $E_{0}$ is the constant polynomial 1 . It is also easy to verify that the following identities hold for $p \in \mathbb{N}$ :

$$
\begin{aligned}
& E_{m-1}^{\langle p\rangle} E_{p-1}=E_{p m-1}, \quad m \in \mathbb{N} ; \\
& \prod_{j=0}^{r-1} E_{p-1}^{\left\langle p^{j}\right\rangle}=E_{p^{r}-1}, r \in \mathbb{N} ; \\
& E_{p^{k}-1} E_{p^{r-k}-1}^{\left\langle p^{k}\right\rangle}=E_{p^{r}-1}, \quad k \in\{0,1, \ldots, r\}, \quad r \in \mathbb{N} .
\end{aligned}
$$

Refinement pairs. In the study of subdivision, an important role is played by so-called refinable functions. For our purposes, the following definition will suffice.

Definition 1.7. We say that $(a, \phi)$ is a $p$-refinement pair if, for some integer $p \geq 2$, sequence $a \in M_{0}(\mathbb{Z})$, and function $\phi \in L^{1}(\mathbb{R}) \backslash\{0\}$, the refinement equation

$$
\phi=\sum_{j \in \mathbb{Z}} a_{j} \phi(p \cdot-j)
$$

is satisfied.

In this case, $\phi$ is said to be a $p$-refinable function. We call $p$ the dilation factor and $a$ the (refinement) mask. We also sometimes call $a$ the mask corresponding to $\phi$ or call $\phi$ the refinable function corresponding to $a$. The polynomial $A=\frac{1}{p} \widetilde{a}$ is called the (refinement) mask symbol.

Remark 1.8. Henceforth, we use the conventions $A=\frac{1}{p} \widetilde{a}, \tilde{A}=\frac{1}{p} \widetilde{a}, A_{k}=\frac{1}{p} \widetilde{a}_{k}$, $B=\frac{1}{p} \vec{b}$, etc., but only for the Roman capitals $A$ and $B$.

From [5, Theorem 2.1, Corollary 2.2, and Theorem 3.1] we have the following necessary conditions for the existence of a $p$-refinement pair.

Theorem 1.9. Suppose $(a, \phi)$ is a p-refinement pair. Then the following assertions hold:

(a) If $(a, \psi)$ is a p-refinement pair, then $\psi=K \phi$ for some real constant $K$.

(b) $A(1)=p^{m}$ for some $m \in \mathbb{Z}_{+}$. 
(c) If, in (b), we have $m \geq 1$, then there exists a function $\psi \in L^{1}(\mathbb{R})$ such that $\left(p^{-m} b, \psi\right)$ is a p-refinement pair, where, with a proper choice of scale, we have $\frac{d^{m}}{d x^{m}} \psi=\phi \quad$ p.p.

(d) $\phi$ is finitely supported, with

$$
\phi(x)=0, \quad x \notin\left[\frac{\downarrow a \downarrow}{p-1}, \frac{\uparrow a \uparrow}{p-1}\right] .
$$

By virtue of points (b) and (c) of Theorem [1.9, we shall henceforth assume, without essential loss of generality, that $A(1)=1$, i.e. $\sum_{j} a_{j}=p$. In this case we have, again from [5], that $\int_{-\infty}^{\infty} \phi(x) d x \neq 0$. We call a refinable function $\phi$ a normalised refinable function if $\int_{-\infty}^{\infty} \phi(x) d x=1$. In this case we call $(a, \phi)$ a normalised $p$-refinement pair.

It can easily be checked that if (1.12) holds, then it follows with $\psi=\phi\left(\cdot+\frac{\downarrow a \downarrow}{p-1}\right)$ and $b_{j}=a_{j+\downarrow a \downarrow}, j \in \mathbb{Z}$, that $\psi=\sum_{j=0}^{\uparrow a \uparrow-\downarrow a \downarrow} b_{j} \psi(p \cdot-j)$. Thus we shall henceforth assume that $\downarrow a \downarrow=0, \uparrow a \uparrow=N$ with $N \in \mathbb{N}$, so that the mask symbol $A$ is a polynomial of degree $N$ with $A(0) \neq 0$.

The following can also be shown (see [6, Lemma 1.9]).

Lemma 1.10. If both $(a, \phi)$ and $(b, \phi)$ are p-refinement pairs, then $a=b$.

A well-known example of a refinable function is provided by $N_{2}$, the cardinal $B$-spline of order 2 , which is given by

$$
N_{2}(x)=\max \{0,1-|1-x|\}, \quad x \in \mathbb{R} .
$$

It is known (see e.g. 8) that, for $p \in \mathbb{Z}, p \geq 2$, the function $N_{2}$ is $p$-refinable with mask $a^{2, p}=p\left[\left(E_{p-1}\right)^{2}\right]$.

Basic results for subdivision. For $p \in \mathbb{Z}, p \geq 2$, suppose that $a, c \in M_{0}(\mathbb{Z})$. Note from (1.8c) that the definition (1.1) is equivalent to $\overparen{S_{a, p} c}=p A \widetilde{c}^{\langle p\rangle}$, so that, by repeated use of (1.8a) and (1.8b),

$$
\widetilde{c^{(r)}}=p A{\widetilde{c^{(r-1)}}}^{\langle p\rangle}=p^{2} A A^{\langle p\rangle}{\overparen{c^{(r-2)}}}^{\langle p-1\rangle}=\cdots=p^{r}\left(\prod_{j=0}^{r-1} A^{\left\langle p^{j}\right\rangle}\right) \widetilde{c}^{\left\langle p^{r}\right\rangle}, \quad r \in \mathbb{N} .
$$

In the special case $c=\delta$, (1.15) becomes

$$
\widetilde{S_{a, p}^{r} \delta}=p^{r} \prod_{j=0}^{r-1} A^{\left\langle p^{j}\right\rangle}, \quad r \in \mathbb{N} .
$$

The link between the subdivision algorithm and refinable functions is borne out by the following result, which is given in [3, Theorem 2.1] for the case $p=2$. It is extended to the general integer multi-dimensional case in [4, Section 3], but we once again use the formulation of [7, Proposition 2] restricted to the univariate case.

Theorem 1.11. Suppose, for $p \in \mathbb{Z}, p \geq 2$, $a \in M_{0}(\mathbb{Z})$, and $c \in l^{\infty}(\mathbb{Z})$, that $\left(S_{a, p}, c\right)$ converges to a function $\Phi_{c}$. Then there exists a unique compactly supported continuous function $\phi$ such that $(a, \phi)$ is a p-refinement pair and such that $\phi$ satisfies the partition of unity property $\sum_{j} \phi(x-j)=1, x \in \mathbb{R}$. Furthermore,

$$
\Phi_{c}=\sum_{j} c_{j} \phi(\cdot-j) .
$$


The next result shows that to check subdivision convergence for all initial sequences in $l^{\infty}(\mathbb{Z})$, it is sufficient to consider the initial sequence $\delta$. The result was first proved for $p=2$ in [3, Proposition 2.2] and the extended multi-dimensional proof is given in [7, Lemma 4], which we once again only state in one-dimensional form.

Theorem 1.12. For $p \in \mathbb{Z}, p \geq 2$, and $a \in M_{0}(\mathbb{Z})$, the subdivision scheme $\left(S_{a, p}, c\right)$ converges for all $c \in l^{\infty}(\mathbb{Z}) \backslash\{0\}$ if and only if $\left(S_{a, p}, \delta\right)$ converges.

We generalise Theorems 1.11 and 1.12 to the case of subsequence convergence in Lemma 3.2 and Theorem 3.3 .

A mask $a$ is said to be a positive mask if it satisfies

$$
a_{j}>0, \quad j \in\{0, \ldots, N\} .
$$

In this special case we have the following subdivision convergence result (see 6. Theorem 2.26]):

Theorem 1.13. Suppose, for dilation factor $p \in \mathbb{Z}, p \geq 2$, that the mask a is positive, the sum rules (1.3) hold for $a$, and $\uparrow a \uparrow \geq p$. Then, for any $c \in \Delta^{\infty}(\mathbb{Z})$, the subdivision scheme $\left(S_{a, p}, c\right)$ converges to the function $\Phi_{c} \in C(\mathbb{R})$ defined by (1.17), where $\phi$ is the normalised p-refinable function corresponding to the refinement mask $a$.

Definition of subsequence convergence. We now return to the study of subsequence convergence in subdivision as discussed in the introduction. We have the following formal definition.

Definition 1.14. For $m \in \mathbb{N}$, we say that the subdivision scheme $\left(S_{a, p}, c\right)$ has $m$-subsequence convergence to $\Phi$ under $K$ if there exists a function $\Phi \in C(\mathbb{R}) \backslash\{0\}$ and a vector $K \in \mathbb{C}^{m}$ satisfying $\sum_{i=0}^{m-1} K_{i}=1$ such that

$$
\max _{i \in \mathbb{Z}_{m}} \sup _{j}\left|K_{i} \Phi\left(\frac{m j+i}{p^{r}}\right)-c_{m j+i}^{(r)}\right| \rightarrow 0, \quad r \rightarrow \infty .
$$

In this case we write $\left(S_{a, p}, c\right) \stackrel{m, K}{\longrightarrow} \Phi$. We call $K$ the subsequence convergence vector $(\mathrm{SCV})$.

Note that the definition (1.18) has the equivalent formulation

$$
\sup _{j}\left|K_{j \bmod m} \Phi\left(\frac{j}{p^{r}}\right)-c_{j}^{(r)}\right| \rightarrow 0, \quad r \rightarrow \infty,
$$

which perhaps expresses the notion more clearly.

Remark 1.15. Subdivision convergence is equivalent to 1-subsequence convergence, since for $m=1$, one obtains $K_{j \bmod m}=K_{0}=1, j \in \mathbb{Z}$, in which case (1.19) becomes (1.2). This shows that $m$-subsequence convergence is a generalisation of the concept of subdivision convergence.

Remark 1.16. Our concept of subsequence convergence must not be confused with the concept called "subconvergence" which is considered in [11, Section 3]. There the convergence of only a subsequence of the iterations of the subdivision scheme are considered, whereas we consider all iterations but take a subsequence of the entries of every iteration. Symbolically, "subconvergence" considers the convergence of $c^{\left(r_{k}\right)}$, where $\left\{r_{k}: k \in \mathbb{Z}_{+}\right\} \subset \mathbb{Z}_{+}$is a strictly increasing sequence, while our concept 
of "subsequence convergence" considers the convergence of $c_{m \cdot+i}^{(r)}$ for appropriately chosen integers $m$ and $i$.

Returning to Example 1.2 we see that it appears that 2-subsequence convergence occurs with $K_{0}=\frac{3}{2}$ and $K_{1}=-\frac{1}{2}$, since $K_{0}=-3 K_{1}$ and $K_{0}+K_{1}$ must equal 1 . This will be shown to indeed be the case by Theorem 4.3 .

\section{Generalised Berg-Plonka factors}

In our analysis, an important role will be played by certain polynomial factors, which are generalisations of the factors considered for the case $p=2$ by [1, 2]. Although we shall not make use of this fact in this paper, these factors correspond to refinable step functions and also play an important role in the analysis of the regularity of a given refinable function (see [6, Sections 2.2, 3.1, and 3.2]).

Definition 2.1. For $p \in \mathbb{Z}, p \geq 2$, we say that a polynomial $P$ is a $p$-GBP (generalised Berg-Plonka) factor if there is an integer $k \in \mathbb{Z}_{+}$such that $P=P_{k}$ can be iteratively obtained as follows:

(1) $P_{0}=E_{p-1}$.

(2) For $l=1,2, \ldots, k, P_{l}$ is obtained by replacing $z$ by $z^{p}$ in $P_{l-1}$ or in a proper polynomial factor of degree at least 1 of $P_{l-1}$.

We say that $P$ is a $p$-GBP factor of level $k$ if $k$ is the smallest integer such that $P=P_{k}$, where $P_{k}$ can be obtained in the algorithm above. For instance, the factor $\frac{1}{2}\left(1+z^{4}\right)$ is a 2 -GBP factor of level 2 , since its shortest possible derivation is

$$
\frac{1}{2}(1+z) \rightarrow \frac{1}{2}\left(1+z^{2}\right) \rightarrow \frac{1}{2}\left(1+z^{4}\right) .
$$

Example 2.2. In Definition 2.1, an important special case is obtained if we form $P_{l}$ by replacing $z$ by $z^{p}$ in $P_{l-1}$ for every $l=1, \ldots, k$, in which case it follows inductively that $P=E_{p-1}^{\left\langle p^{k}\right\rangle}$. If a $p$-GBP factor is not of this special form, we shall call it a nontrivial $p$-GBP factor.

We show some nontrivial 2-GBP factors in Figure 2.1, which depicts the derivation of all 2-GBP factors up to level 2 and the 2-GBP factors of level 3 with real coefficients. Although we do not show the calculations here, it is interesting to note that there are an additional eighteen 2-GBP factors (each with at least one complex coefficient) of level 3, yielding a total of twenty-six 2-GBP factors of level at most 3.

Remark 2.3. GBP factors have a useful equivalent formulation, which was noted in the proof of [1, Theorem 3.4] for the case $p=2$. Namely, $P$ is a $p$-GBP factor if and only if there are an integer $k \in \mathbb{Z}_{+}$and polynomials $q_{l}, r_{l}: l \in\{0, \ldots, k\}$ with $\operatorname{deg}\left(r_{l}\right) \geq 1, l \in\{0, \ldots, k\}$, such that the following identities hold:

$$
\begin{aligned}
q_{0} r_{0} & =E_{p-1} ; \\
q_{l} r_{l} & =q_{l-1} r_{l-1}^{\langle p\rangle}, \quad l=1,2, \ldots, k ; \\
q_{k} r_{k} & =P .
\end{aligned}
$$

To see the equivalence of this definition, note that $q_{l} r_{l}=P_{l}$ for $l=0,1, \ldots, k$, with $r_{l-1}$ representing the polynomial factor of $P_{l-1}$ in which $z$ is replaced by $z^{p}$ 


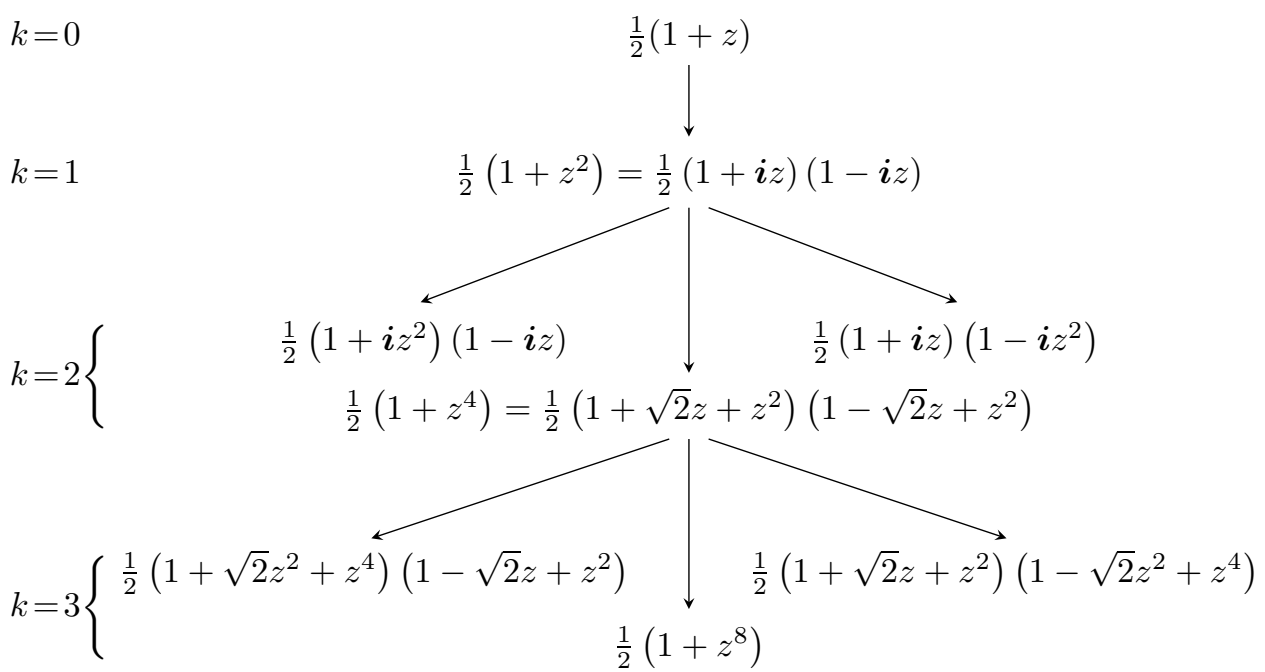

Figure 2.1. A graphic illustration showing the derivation of the 2GBP factors up to level 2 and those of level 3 with real coefficients. There are eighteen other 2-GBP factors of level 3.

for $l=1,2, \ldots, k$. Also note that, since $E_{p-1}(1)=1$ and

$$
q_{l}(1) r_{l}(1)=q_{l-1}(1) r_{l-1}^{\langle p\rangle}(1)=q_{l-1}(1) r_{l-1}(1), \quad l=1,2, \ldots, k,
$$

it follows inductively that $P_{l}(1)=1$ for $l=0,1, \ldots, k$. Thus without loss of generality we can always choose the $q_{l}, r_{l}$ such that

$$
q_{l}(1)=r_{l}(1)=1, \quad l=0,1, \ldots, k .
$$

The next lemma establishes some useful properties of GBP factors, which we shall employ later.

Lemma 2.4. For $p \in \mathbb{Z}, p \geq 2$, suppose that $P$ is a $p$-GBP factor of level $k$. Then $P$ can be expressed in the form

$$
P=E_{p-1} \frac{R^{\langle p\rangle}}{R},
$$

where $R$ is polynomial satisfying $R(1)=1$. Furthermore, the function $W$, given by $W=E_{p^{k}-1} / R$, is a polynomial satisfying $W(1)=1$.

Proof. By rewriting (2.1b) as $q_{l}=q_{l-1} \frac{r_{l-1}^{\langle p\rangle}}{r_{l}}$, we obtain from (2.1a) (2.1c) that

$$
P=q_{k-1} r_{k-1}^{\langle p\rangle}=q_{k-2} r_{k-2}^{\langle p\rangle} \frac{r_{k-1}^{\langle p\rangle}}{r_{k-1}}=\cdots=q_{0} r_{0}^{\langle p\rangle} \prod_{l=1}^{k-1} \frac{r_{l}^{\langle p\rangle}}{r_{l}}=E_{p-1} \prod_{l=0}^{k-1} \frac{r_{l}^{\langle p\rangle}}{r_{l}} .
$$

Defining $R$ by

$$
R=\prod_{l=0}^{k-1} r_{l}
$$

we see that $R$ is a polynomial, while (2.4) shows that (2.3) holds. From the assumption (2.2) it follows that $R(1)=1$. 
We also have, by consecutively using (1.11b), (2.1a), (1.8b), (1.8a), (2.1b), and (2.5), that

$$
\begin{aligned}
E_{p^{k}-1} & =\prod_{l=0}^{k-1} E_{p-1}^{\left\langle p^{l}\right\rangle}=\prod_{l=0}^{k-1} q_{0}^{\left\langle p^{l}\right\rangle} r_{0}^{\left\langle p^{l}\right\rangle}=r_{0} q_{0}^{\left\langle p^{k-1}\right\rangle} \prod_{l=0}^{k-2} q_{0}^{\left\langle p^{l}\right\rangle} r_{0}^{\left\langle p^{l+1}\right\rangle} \\
& =r_{0} q_{0}^{\left\langle p^{k-1}\right\rangle} \prod_{l=0}^{k-2}\left(q_{0} r_{0}^{\langle p\rangle}\right)^{\left\langle p^{l}\right\rangle}=r_{0} q_{0}^{\left\langle p^{k-1}\right\rangle} \prod_{l=0}^{k-2}\left(q_{1} r_{1}\right)^{\left\langle p^{l}\right\rangle} \\
& =r_{0} q_{0}^{\left\langle p^{k-1}\right\rangle} r_{1} q_{1}^{\left\langle p^{k-2}\right\rangle} \prod_{l=0}^{k-3} q_{1}^{\left\langle p^{l}\right\rangle} r_{1}^{\left\langle p^{l+1}\right\rangle} \\
& =r_{0} q_{0}^{\left\langle p^{k-1}\right\rangle} r_{1} q_{1}^{\left\langle p^{k-2}\right\rangle} \prod_{l=0}^{k-3}\left(q_{2} r_{2}\right)^{\left\langle p^{l}\right\rangle} \\
& =\cdots=\prod_{l=0}^{k-1} r_{l} q_{l}^{\left\langle p^{k-1-l}\right\rangle}=R \prod_{l=0}^{k-1} q_{l}^{\left\langle p^{k-1-l}\right\rangle},
\end{aligned}
$$

so that $W=E_{p^{k}-1} / R=\prod_{l=0}^{k-1} q_{l}^{\left\langle p^{k-1-l}\right\rangle}$ is a polynomial. To complete the proof of the lemma, we observe that $W(1)=E_{p^{k}-1}(1) / R(1)=1$.

Remark 2.5. In Lemma 2.4, for the special case $P=E_{p-1}^{\left\langle p^{k}\right\rangle}$ which was mentioned in Example 2.2, we have $q_{l}=E_{0}$ for $l=0, \ldots, k$ in (2.1a) (2.1c), so that

$$
r_{l}=E_{p-1}^{\left\langle p^{l}\right\rangle}, \quad l=0, \ldots, k,
$$

which by (2.5) and (1.11b) yields $R=E_{p^{k}-1}$, which yields $W=E_{p^{k}-1} / R=E_{0}$.

\section{BASIC PROPERTIES OF SUbSEQUENCE CONVERGENCE}

We proceed to obtain some basic properties related to subsequence convergence.

Lemma 3.1. Suppose, for $a, c \in M_{0}(\mathbb{Z})$ and $p \in \mathbb{Z}, p \geq 2$, that $\left(S_{a, p}, c\right) \stackrel{m, K}{\longrightarrow} \Phi$. Then the following statements are true:

(a) $\Phi$ is compactly supported, with

$$
\Phi(x)=0, \quad x \notin\left(\downarrow c \downarrow, \frac{\uparrow a \uparrow}{p-1}+\uparrow c \uparrow\right) .
$$

(b) If $\left(S_{a, p}, c\right) \stackrel{m, \tilde{K}}{\longrightarrow} \tilde{\Phi}$, then $\Phi=\tilde{\Phi}$ and $K=\tilde{K}$.

(c) For any $n \in \mathbb{N},\left(S_{a, p}, c\right) \stackrel{m n, K^{(n)}}{\longrightarrow} n \Phi$, where the vector $K^{(n)} \in \mathbb{C}^{m n}$ is given by $\left(K^{(n)}\right)_{i}=\frac{1}{n} K_{i \bmod m}, i \in \mathbb{Z}_{m n}$.

(d) If $m=n l$, with $l, n \in \mathbb{N}, n \geq 2$, then one has $\left(S_{a, p}, c\right) \stackrel{l, \tilde{K}}{\longrightarrow} \frac{1}{n} \Phi$ if and only if $K_{i}=K_{i \bmod l}, i \in \mathbb{Z}_{m}$. In this case, $\tilde{K}_{i}=n K_{i}, i \in \mathbb{Z}_{l}$.

Proof. (a) From (1.15), (1.6), and (1.7), after recalling also $\downarrow a \downarrow=0$, we obtain, for $r \in \mathbb{N}$,

$$
\uparrow c^{(r)} \uparrow=\sum_{j=0}^{r-1} p^{j} \uparrow a \uparrow+p^{r} \uparrow c \uparrow=\frac{p^{r}-1}{p-1} \uparrow a \uparrow+p^{r} \uparrow c \uparrow
$$


and

$$
\downarrow c^{(r)} \downarrow=\sum_{j=0}^{r-1} p^{j} \downarrow a \downarrow+p^{r} \downarrow c \downarrow=p^{r} \downarrow c \downarrow .
$$

Suppose now that $x \notin\left[\downarrow c \downarrow, \frac{\uparrow a \uparrow}{p-1}+\uparrow c \uparrow\right]$ and let the sequence $\left\{j_{r}: r \in \mathbb{Z}_{+}\right\} \subset \mathbb{R}$ be such that $\frac{j_{r}}{p^{r}} \rightarrow x$ as $r \rightarrow \infty$. Then there is an integer $R$ such that $\frac{j_{r}}{p^{r}} \notin$ $\left[\downarrow c \downarrow, \frac{\uparrow a \uparrow}{p-1}+\uparrow c \uparrow\right]$ if $r \geq R$, so that also $j_{r} \notin\left[p^{r} \downarrow c \downarrow, \frac{p^{r}-1}{p-1} \uparrow a \uparrow+p^{r} \uparrow c \uparrow\right]$ whenever $r \geq R$, which, by (3.2a) and (3.2b), yields $c_{j_{r}}^{(r)}=0, r \geq R$. But then (1.18), together with the fact that $\Phi \in C(\mathbb{R})$, yields the desired compact support property (3.1) of $\Phi$.

(b) Suppose that $\left(S_{a, p}, c\right) \stackrel{m, \tilde{K}}{\longrightarrow} \tilde{\Phi}$. It follows from (1.19) by the triangle inequality that

$$
\sup _{j}\left|K_{j \bmod m} \Phi\left(\frac{j}{p^{r}}\right)-\tilde{K}_{j \bmod m} \tilde{\Phi}\left(\frac{j}{p^{r}}\right)\right| \rightarrow 0, \quad r \rightarrow \infty,
$$

from which we conclude by the continuity of $\Phi$ and $\tilde{\Phi}$, together with the fact that the set $\left\{\frac{j}{p^{r}}: j \in \mathbb{Z}, r \in \mathbb{Z}_{+}\right\}$is dense in $\mathbb{R}$, that $K_{i} \Phi=\tilde{K}_{i} \tilde{\Phi}, i \in \mathbb{Z}_{m}$. Together with the identities $\sum_{i=0}^{m-1} K_{i}=1=\sum_{i=0}^{m-1} \tilde{K}_{i}$, this yields

$$
\Phi=\sum_{i \in \mathbb{Z}_{m}} K_{i} \Phi=\sum_{i \in \mathbb{Z}_{m}} \tilde{K}_{i} \tilde{\Phi}=\tilde{\Phi} .
$$

Since $\Phi \neq 0$ by definition, there exists an $x_{0} \in \mathbb{R}$ such that $\Phi\left(x_{0}\right) \neq 0$. Furthermore, since

we obtain $K_{i}=\tilde{K}_{i}, i \in \mathbb{Z}_{m}$.

$$
K_{i} \Phi\left(x_{0}\right)=\tilde{K}_{i} \tilde{\Phi}\left(x_{0}\right)=\tilde{K}_{i} \Phi\left(x_{0}\right), \quad i \in \mathbb{Z}_{m},
$$

(c) Suppose $n \in \mathbb{N}$. Then we obtain, for $r \in \mathbb{Z}_{+}$,

$$
\begin{aligned}
& \max _{i \in \mathbb{Z}_{m n}} \sup _{j}\left|\frac{1}{n} K_{i \bmod m} n \Phi\left(\frac{m n j+i}{p^{r}}\right)-c_{m n j+i}^{(r)}\right| \\
& =\max _{i \in \mathbb{Z}_{m}} \max _{k \in \mathbb{Z}_{n}} \sup _{j}\left|K_{(k m+i) \bmod m} \Phi\left(\frac{m(n j+k)+i}{p^{r}}\right)-c_{m(n j+k)+i}^{(r)}\right| \\
& =\max _{i \in \mathbb{Z}_{m}} \sup _{j}\left|K_{i} \Phi\left(\frac{m j+i}{p^{r}}\right)-c_{m j+i}^{(r)}\right|,
\end{aligned}
$$

which yields the desired result by (1.18) after noting also that

$$
\sum_{i=0}^{m n-1} \frac{1}{n} K_{i \bmod m}=\sum_{i=0}^{m-1} \sum_{k=0}^{n-1} \frac{1}{n} K_{(i+m k) \bmod m}=\sum_{i=0}^{m-1} K_{i}=1 .
$$

(d) Assume $m=n l$, with $l, n \in \mathbb{N}, n \geq 2$. Now first suppose that $\left(S_{a, p}, c\right) \stackrel{l, \tilde{K}}{\longrightarrow}$ $\frac{1}{n} \Phi$. Then by the result (c), we have $\left(S_{a, p}, c\right) \stackrel{m, K^{\prime}}{\longrightarrow} \Phi$, where $K_{i}^{\prime}=\frac{1}{n} \tilde{K}_{i \bmod l}, i \in$ $\mathbb{Z}_{m}$. Now from (b) we have $K=K^{\prime}$, so that $K_{i}=\frac{1}{n} \tilde{K}_{i \bmod l}, i \in \mathbb{Z}_{m}$, from which we obtain

$$
K_{i \bmod l}=\frac{1}{n} \tilde{K}_{(i \bmod l) \bmod l}=\frac{1}{n} \tilde{K}_{i \bmod l}=K_{i}, \quad i \in \mathbb{Z}_{m} .
$$


Conversely, suppose that $K_{i}=K_{i \bmod l}, i \in \mathbb{Z}_{m}$, which has the equivalent formulation $K_{i}=K_{i+l k}, i \in \mathbb{Z}_{l}, k \in \mathbb{Z}_{n}$. From this identity, together with $m=l n$ and the assumed $m$-subsequence convergence of $\left(S_{a, p}, c\right)$ to $\Phi$, we obtain

$$
\begin{aligned}
& \max _{i \in \mathbb{Z}_{l}} \sup _{j}\left|n K_{i} \frac{1}{n} \Phi\left(\frac{l j+i}{p^{r}}\right)-c_{j l+i}^{(r)}\right| \\
& =\max _{i \in \mathbb{Z}_{l}} \sup _{j} \max _{k \in \mathbb{Z}_{n}}\left|K_{i} \Phi\left(\frac{l(n j+k)+i}{p^{r}}\right)-c_{l(n j+k)+i}^{(r)}\right| \\
& =\max _{i \in \mathbb{Z}_{l}} \max _{k \in \mathbb{Z}_{n}} \sup _{j}\left|K_{i+l k} \Phi\left(\frac{m j+l k+i}{p^{r}}\right)-c_{m j+l k+i}^{(r)}\right| \\
& =\max _{i \in \mathbb{Z}_{m}} \sup _{j}\left|K_{i} \Phi\left(\frac{m j+i}{p^{r}}\right)-c_{m j+i}^{(r)}\right| \rightarrow 0, \quad r \rightarrow \infty .
\end{aligned}
$$

This, together with the identity

$$
\sum_{i=0}^{l-1} n K_{i}=\sum_{i=0}^{l-1} \sum_{k=0}^{n-1} K_{i}=\sum_{i=0}^{l-1} \sum_{k=0}^{n-1} K_{i+l k}=\sum_{i=0}^{m-1} K_{i}=1,
$$

yields $\left(S_{a, p}, c\right) \stackrel{l, \tilde{K}}{\longrightarrow} \frac{1}{n} \Phi$, with $\tilde{K}_{i}=n K_{i}, i \in \mathbb{Z}_{l}$, thereby completing the proof of the lemma.

The next lemma shows that, as in the case of normal subdivision convergence, it is sufficient to consider the initial sequence $\delta$.

Lemma 3.2. If $\left(S_{a, p}, \delta\right) \stackrel{m, K}{\longrightarrow} \phi$, then, for any initial sequence $c \in l^{\infty}(\mathbb{Z}) \backslash\{0\}$, one has $\left(S_{a, p}, c\right) \stackrel{m, K}{\longrightarrow} \Phi_{c}$, where $\Phi_{c}$ and $\phi$ are related by (1.17).

Proof. As before, let $N=\uparrow a \uparrow$. From the assumption $\left(S_{a, p}, \delta\right) \stackrel{m, K}{\longrightarrow} \phi$, it can be shown inductively that

$$
\left(S_{a}^{r} c\right)_{j}=\sum_{l}\left(S_{a}^{r} \delta\right)_{j-p^{r} l} c_{l}, \quad j \in \mathbb{Z}, \quad r \in \mathbb{Z}_{+}, \quad c \in M(\mathbb{Z}),
$$

with the inductive step following from the definition (1.1).

Given any initial sequence $c \in l^{\infty}(\mathbb{Z})$ in the subdivision algorithm, we obtain by use of (1.17) and (3.3) that the identity

$$
K_{i} \Phi_{c}\left(\frac{m j+i}{p^{r}}\right)-\left(S_{a}^{r} c\right)_{m j+i}=\sum_{l} c_{l}\left(K_{i} \phi\left(\frac{m j+i}{p^{r}}-l\right)-\left(S_{a}^{r} \delta\right)_{m j+i-p^{r} l}\right)
$$

holds for $j \in \mathbb{Z}, r \in \mathbb{Z}_{+}$, and $i \in\{0, \ldots, m-1\}$.

If we define, for $r \in \mathbb{Z}_{+}, j \in \mathbb{Z}$, the constants $\mu_{r, j}, \nu_{r, j} \in \mathbb{Z}$ by

$$
\mu_{r, j}=\left\lceil\frac{m j}{p^{r}}\right\rceil-N \quad \text { and } \quad \nu_{r, j}=\left\lfloor\frac{m j+m-1}{p^{r}}\right\rfloor,
$$

it follows from (1.16), (1.6), (1.7), and Lemma 3.1(a) applied to the initial sequence $\delta$, after recalling also $\uparrow \delta \uparrow=0=\downarrow \delta \downarrow$ and $\uparrow a \uparrow=N$, that the equalities

$$
\phi\left(\frac{m j+i}{p^{r}}-l\right)=0=\left(S_{a}^{r} \delta\right)_{m j+i-p^{r} l}, \quad l \in \mathbb{Z} \backslash\left\{\mu_{r, j}, \ldots, \nu_{r, j}\right\},
$$


hold for $i \in\{0, \ldots, m-1\}$. Note from (3.5) that we have $0 \leq \nu_{r, j}-\mu_{r, j} \leq N$ for $j \in \mathbb{Z}$ if $r>\log _{p}(m-1)$, from which, together with (3.4), (3.6), and (1.18) applied to the initial sequence $\delta$, we find that, for any $\varepsilon>0$, the inequalities

$$
\begin{aligned}
\left|K_{i} \Phi_{c}\left(\frac{m j+i}{p^{r}}\right)-\left(S_{a}^{r} c\right)_{m j+i}\right| & \leq \sum_{l=\mu_{r, j}}^{\nu_{r, j}}\left|c_{l}\right|\left|K_{i} \phi\left(\frac{m j+i}{p^{r}}-l\right)-\left(S_{a}^{r} \delta\right)_{m j+i-p^{r} l}\right| \\
& \leq \sum_{l=\mu_{r, j}}^{\nu_{r, j}}\left|c_{l}\right| \varepsilon \leq(N+1)\|c\|_{\infty} \varepsilon
\end{aligned}
$$

hold for $j \in \mathbb{Z}$ and $i \in\{0, \ldots, m-1\}$ by taking $r$ large enough. This shows that $\left(S_{a, p}, c\right) \stackrel{m, K}{\longrightarrow} \Phi_{c}$.

The next result extends another well-known property of standard subdivision convergence to the case of subsequence convergence, namely that the limit function must be refinable if one starts with the delta sequence.

Theorem 3.3. If, for some $k \in \mathbb{Z}_{+}$, the subdivision scheme $\left(S_{a, p}, \delta\right)$ has $p^{k}$ subsequence convergence with limit function $\phi$, then $(a, \phi)$ is a p-refinement pair.

Proof. By application of (1.16), we obtain

$$
\overparen{S_{a}^{r+1} \delta}=p^{r+1} \prod_{j=0}^{r} A^{\left\langle p^{j}\right\rangle}=p A^{\left\langle p^{r}\right\rangle} \overparen{S_{a}^{r} \delta}, \quad r \in \mathbb{Z}_{+}
$$

which by $1.8 \mathrm{c}$ is equivalent to

$$
\left(S_{a}^{r+1} \delta\right)_{j}=\sum_{l} a_{l}\left(S_{a}^{r} \delta\right)_{j-p^{r} l}, \quad j \in \mathbb{Z}, \quad r \in \mathbb{Z}_{+}
$$

By assumption we have $\left(S_{a, p}, \delta\right) \stackrel{p^{k}, K}{\longrightarrow} \phi$ for some $K \in \mathbb{C}^{p^{k}}$. Since $\sum_{i=0}^{p^{k}-1} K_{i}=1$, there is an index $i \in\left\{0, \ldots, p^{k}-1\right\}$ such that $K_{i} \neq 0$. Let now $x \in \mathbb{R}$ be fixed and choose a sequence $\left\{j_{r}: r \in \mathbb{Z}_{+}\right\}$such that $\frac{j_{r}}{p^{r}} \rightarrow p^{1-k} x$ as $r \rightarrow \infty$ and let $\varepsilon>0$ be given.

We have, by use of (3.7), that, for $r \in \mathbb{Z}_{+}, r \geq k$,

$$
\begin{aligned}
& K_{i}\left(\phi\left(\frac{p^{k} j_{r}+i}{p^{r+1}}\right)-\sum_{l} a_{l} \phi\left(\frac{p^{k} j_{r}+i}{p^{r}}-l\right)\right) \\
& =\left(K_{i} \phi\left(\frac{p^{k} j_{r}+i}{p^{r+1}}\right)-\left(S_{a}^{r+1} \delta\right)_{p^{k} j_{r}+i}\right) \\
& +\sum_{l} a_{l}\left(\left(S_{a}^{r} \delta\right)_{p^{k}\left(j_{r}-p^{r-k} l\right)+i}-K_{i} \phi\left(\frac{p^{k}\left(j_{r}-p^{r-k} l\right)+i}{p^{r}}\right)\right) .
\end{aligned}
$$

Together with the definition (1.18) of subsequence convergence, this yields the existence of an integer $R \geq k$ such that

$$
\left|K_{i}\right|\left|\phi\left(\frac{p^{k} j_{r}+i}{p^{r+1}}\right)-\sum_{l} a_{l} \phi\left(\frac{p^{k} j_{r}+i}{p^{r}}-l\right)\right|<\varepsilon+\sum_{l}\left|a_{l}\right| \varepsilon=C \varepsilon, \quad r \geq R,
$$


where

$$
C=1+\sum_{l}\left|a_{l}\right|<\infty
$$

According to Lemma 3.1(a), $\phi$ is compactly supported, so that $\phi \in C(\mathbb{R})$ implies that $\phi$ is uniformly continuous on $\mathbb{R}$. Hence there is a $\tau>0$ such that

$$
|\phi(x)-\phi(y)|<\varepsilon, \quad x, y \in \mathbb{R}, \quad|x-y|<\tau .
$$

Since $\lim _{r \rightarrow \infty} \frac{p^{k} j_{r}+i}{p^{r+1}}=\lim _{r \rightarrow \infty}\left(p^{k-1} \frac{j_{r}}{p^{r}}+\frac{i}{p^{r+1}}\right)=x$, there is an integer $R^{\prime}$ such that $r \geq R^{\prime}$ implies $\left|\frac{p^{k} j_{r}+i}{p^{r+1}}-x\right|<\frac{\tau}{p}$. Thus

$$
\left|\left(\frac{p^{k} j_{r}+i}{p^{r}}-l\right)-(p x-l)\right|=p\left|\frac{p^{k} j_{r}+i}{p^{r+1}}-x\right|<\tau, \quad l \in \mathbb{Z}, \quad r \geq R^{\prime} .
$$

Since also $\frac{\tau}{p}<\tau$, we now have, by (3.10), for $r \geq R^{\prime}$,

$$
\left|\phi\left(\frac{p^{k} j_{r}+i}{p^{r+1}}\right)-\phi(x)\right|<\varepsilon
$$

and

$$
\left|\phi\left(\frac{p^{k} j_{r}+i}{p^{r}}-l\right)-\phi(p x-l)\right|<\varepsilon, \quad l \in \mathbb{Z} .
$$

If we take $r \geq \max \left\{R, R^{\prime}\right\}$, we have from (3.8), (3.9), (3.11), and (3.12) that

$$
\begin{aligned}
& \left|\phi(x)-\sum_{l} a_{l} \phi(p x-l)\right| \\
& =\mid \phi(x)-\phi\left(\frac{p^{k} j_{r}+i}{p^{r+1}}\right)+\phi\left(\frac{p^{k} j_{r}+i}{p^{r+1}}\right)-\sum_{l} a_{l} \phi\left(\frac{p^{k} j_{r}+i}{p^{r}}-l\right) \\
& +\sum_{l} a_{l}\left(\phi\left(\frac{p^{k} j_{r}+i}{p^{r}}-l\right)-\phi(p x-l)\right) \mid \\
& \leq\left|\phi(x)-\phi\left(\frac{p^{k} j_{r}+i}{p^{r+1}}\right)\right|+\left|\phi\left(\frac{p^{k} j_{r}+i}{p^{r+1}}\right)-\sum_{l} a_{l} \phi\left(\frac{p^{k} j_{r}+i}{p^{r}}-l\right)\right| \\
& +\sum_{l}\left|a_{l}\right|\left|\phi\left(\frac{p^{k} j_{r}+i}{p^{r}}-l\right)-\phi(p x-l)\right| \\
& <\varepsilon+\frac{C \varepsilon}{\left|K_{i}\right|}+\sum_{l}\left|a_{l}\right| \varepsilon=C \varepsilon\left(1+\frac{1}{\left|K_{i}\right|}\right) .
\end{aligned}
$$

Since $\varepsilon>0$ is arbitrary, we deduce that $\phi(x)=\sum_{l} a_{l} \phi(p x-l)$ for any given $x \in \mathbb{R}$, which, together with the compact support of $\phi$ and the fact that $\phi$ is not identically zero, yields the desired result.

\section{Sufficient COnditions For Subsequence CONVERGEnCE}

One trivial set of conditions which yields subsequence convergence occurs in the case of "stretched" masks, as formulated in the following theorem. 
Theorem 4.1. For $p \in \mathbb{N}, p \geq 2$, and $m \in \mathbb{N}$, if the masks a and $\tilde{a}$ are related by

$$
a_{j m+l}=\delta_{l} \tilde{a}_{j}, \quad j \in \mathbb{Z}, \quad l \in \mathbb{Z}_{m},
$$

and the subdivision algorithm $\left(S_{\tilde{a}, p}, \delta\right)$ converges to a function $\phi$, then $\left(S_{a, p}, \delta\right) \stackrel{m, K}{\longrightarrow}$ $\phi(\dot{\bar{m}})$, with $K \in \mathbb{C}^{m}$ given by $K_{i}=\delta_{i}, i \in \mathbb{Z}_{m}$.

Proof. Set, as usual, $c^{(r)}=S_{a, p}^{r} \delta$ and $\tilde{c}^{(r)}=S_{\tilde{a}, p}^{r} \delta$. We show by induction that

$$
c_{j m+l}^{(r)}=\delta_{l} \tilde{c}_{j}^{(r)}, \quad j \in \mathbb{Z}, \quad l \in \mathbb{Z}_{m},
$$

for $r \in \mathbb{Z}_{+}$. For $r=0$ we obtain, for $l \in \mathbb{Z}_{m}, c_{j m+l}^{(0)}=\delta_{j m+l}=\delta_{l} \delta_{j}=\delta_{l} \tilde{c}_{j}^{(0)}$. Supposing now that (4.1) holds for a given $r \in \mathbb{Z}_{+}$, we obtain, for $j \in \mathbb{Z}$ and $l \in \mathbb{Z}_{m}$

$$
\begin{aligned}
c_{j m+l}^{(r+1)} & =\sum_{k} a_{j m+l-p k} c_{k}^{(r)}=\sum_{k} \sum_{n=0}^{m-1} a_{j m+l-p(k m+n)} c_{k m+n}^{(r)} \\
& =\sum_{k} \sum_{n=0}^{m-1} a_{(j-p k) m+l-p n} \delta_{n} \tilde{c}_{k}^{(r)}=\sum_{k} a_{(j-p k) m+l} \tilde{c}_{k}^{(r)} \\
& =\sum_{k} \delta_{l} \tilde{a}_{j-p k} \tilde{c}_{k}^{(r)}=\delta_{l} \tilde{c}_{j}^{(r+1)},
\end{aligned}
$$

which completes the inductive step.

Denoting $\phi\left(\frac{\dot{m}}{m}\right)$ by $f$, we obtain, from (4.1) and the convergence of $\left(S_{\tilde{a}, p}, \delta\right)$ to $\phi$, that

$$
\begin{aligned}
\max _{i \in \mathbb{Z}_{m}} \sup _{j}\left|\delta_{i} f\left(\frac{m j+i}{p^{r}}\right)-c_{m j+i}^{(r)}\right| & =\max _{i \in \mathbb{Z}_{m}} \sup _{j}\left|\delta_{i}\left(f\left(\frac{m j+i}{p^{r}}\right)-\tilde{c}_{j}^{(r)}\right)\right| \\
& =\sup _{j}\left|f\left(\frac{m j}{p^{r}}\right)-\tilde{c}_{j}^{(r)}\right| \\
& =\sup _{j}\left|\phi\left(\frac{j}{p^{r}}\right)-\tilde{c}_{j}^{(r)}\right| \rightarrow 0, \quad r \rightarrow \infty,
\end{aligned}
$$

which completes the proof of the theorem.

Example 4.2. In order to illustrate Theorem 4.1 for $p=2$, consider the mask with symbol

$$
A(z)=\frac{1}{4}\left(1+z^{3}\right)^{2}, \quad z \in \mathbb{C},
$$

which was also considered in [10, Example 2.1]. In the notation of Theorem 4.1, we have here $m=3$ and $\tilde{A}=\left(E_{1}\right)^{2}$. Since $\left(S_{\tilde{a}, 2}, \delta\right)$ converges to $N_{2}$, Theorem 4.1 tells us that $\left(S_{a, 2}, \delta\right)$ has 3 -subsequence convergence to $N_{2}(\dot{\overline{3}})$, i.e. the linear $B$-spline with knots $\{0,3,6\}$, with subsequence convergence constants $K_{0}=1, K_{1}=K_{2}=0$. This is illustrated in Figure 4.1 .

This elementary case is well known (see e.g. [10]), although the notion of subsequence convergence has not, to our knowledge, been formally defined before. We are of course interested in less elementary cases, as for instance in Example 1.2 , which is not covered by Theorem 4.1. In order to derive such less trivial sufficient conditions for subsequence convergence to occur, we shall make use of the GBP-factors of Section 2. This leads us to the following result. 


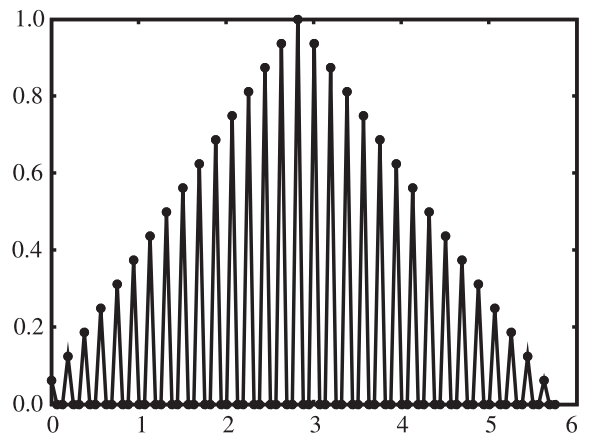

(a)

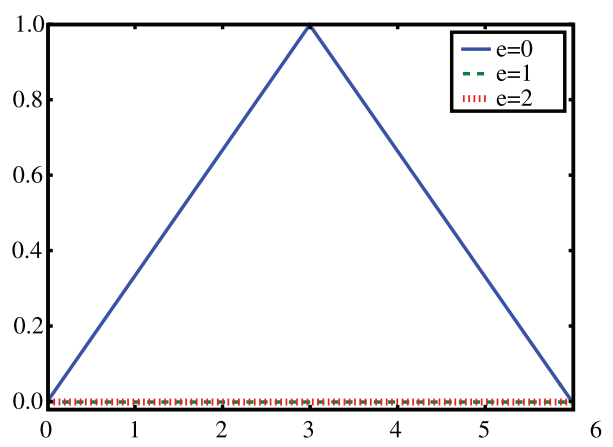

(b)

Figure 4.1. Plots of (a) $S_{a}^{4} \delta$ and (b) $\left(S_{a}^{8} \delta\right)_{3 \cdot+e}, e=0,1,2$, in Example 4.2 ,

Theorem 4.3. For dilation factor $p \in \mathbb{Z}, p \geq 2$, suppose the mask symbol $A$ satisfies $A=P B$, where $P$ is a $p$-GBP factor of level $k$ and $B$ is some polynomial. Let the polynomials $R, W$ be as in Lemma 2.4 and let the mask symbol $\tilde{A}$ be defined by

$$
\tilde{A}=E_{p-1} B
$$

Then, if the subdivision algorithm $\left(S_{\tilde{a}, p}, c\right)$ converges to $\tilde{\Phi}$, we have $\left(S_{a, p}, c\right) \stackrel{p^{k}, K}{\longrightarrow}$ $\Phi$, with the function $\Phi$ defined by

$$
\Phi=p^{k} \sum_{j}[R]_{j} \tilde{\Phi}(\cdot-j)
$$

and $K \in \mathbb{C}^{p^{k}}$ given by

$$
K_{i}=\sum_{l}\left[W \prod_{j=0}^{k-1} B^{\left\langle p^{j}\right\rangle}\right]_{i-p^{k} l}, \quad i=0,1, \ldots, p^{k}-1 .
$$

Proof. By Lemma 3.2, it is sufficient to consider the initial sequence $c=\delta$; hence, letting $\tilde{\phi}$ denote the limit of the subdivision scheme $\left(S_{\tilde{a}, p}, \delta\right)$, we have to show that $\left(S_{a, p}, \delta\right) \stackrel{p^{k}, K}{\longrightarrow} \phi$, where $\phi$ is defined, in accordance with (4.3), by

$$
\phi=p^{k} \sum_{j}[R]_{j} \tilde{\phi}(\cdot-j)
$$

while $K$ is as in (4.4).

From (1.16) we obtain

$$
\widetilde{S_{\tilde{a}}^{r} \delta}=p^{r} \prod_{j=0}^{r-1} \tilde{A}^{\left\langle p^{j}\right\rangle}, \quad r \in \mathbb{N} .
$$


By using (1.16), (2.3), (1.8b), (1.8a), (1.11b), and (4.2), we now obtain, for $r \in$ $\mathbb{N}, r>k$,

$$
\begin{aligned}
\widetilde{S_{a}^{r} \delta} & =p^{r} \prod_{j=0}^{r-1} E_{p-1}^{\left\langle p^{j}\right\rangle} \frac{R^{\left\langle p^{j+1}\right\rangle}}{R^{\left\langle p^{j}\right\rangle}} B^{\left\langle p^{j}\right\rangle} \\
& =p^{r} \frac{R^{\left\langle p^{r}\right\rangle}}{R}\left(\prod_{j=0}^{k-1} E_{p-1}^{\left\langle p^{j}\right\rangle} B^{\left\langle p^{j}\right\rangle}\right)\left(\prod_{j=k}^{r-1} E_{p-1}^{\left\langle p^{j}\right\rangle} B^{\left\langle p^{j}\right\rangle}\right) \\
& =p^{r} \frac{R^{\left\langle p^{r}\right\rangle}}{R}\left(\prod_{j=0}^{k-1} E_{p-1}^{\left\langle p^{j}\right\rangle} B^{\left\langle p^{j}\right\rangle}\right)\left(\prod_{j=0}^{r-k-1} E_{p-1}^{\left\langle p^{j+k}\right\rangle} B^{\left\langle p^{j+k}\right\rangle}\right) \\
& =p^{r} \frac{R^{\left\langle p^{r}\right\rangle}}{R}\left(\prod_{j=0}^{k-1} E_{p-1}^{\left\langle p^{j}\right\rangle} B^{\left\langle p^{j}\right\rangle}\right)\left(\prod_{j=0}^{r-k-1} E_{p-1}^{\left\langle p^{j}\right\rangle} B^{\left\langle p^{j}\right\rangle}\right) \\
& =p^{r} \frac{E_{p^{k}-1}}{R}\left(\prod_{j=0}^{k-1} B^{\left\langle p^{j}\right\rangle}\right)\left(R^{\left\langle p^{r-k}\right\rangle} \prod_{j=0}^{r-k-1} \tilde{A}^{\left\langle p^{j}\right\rangle}\right) \\
& =U V_{r}^{\left\langle p^{k}\right\rangle},
\end{aligned}
$$

where, by noting (4.6) and the definition of $W$, the polynomials $U$ and $V$ are defined by

$$
\begin{gathered}
U=p^{k} W \prod_{j=0}^{k-1} B^{\left\langle p^{j}\right\rangle} \\
V_{r}=R^{\left\langle p^{r-k}\right\rangle} \overparen{S_{\tilde{a}}^{r-k} \delta} .
\end{gathered}
$$

and

We thus find, by also using (1.8c), that, for $j \in \mathbb{Z}$ and $i \in\left\{0,1, \ldots, p^{k}-1\right\}$,

$$
\begin{aligned}
\left(S_{a}^{r} \delta\right)_{p^{k} j+i} & =\sum_{l}[U]_{p^{k}(j-l)+i}\left[V_{r}\right]_{l} \\
& =\sum_{l}[U]_{i-p^{k} l}\left[V_{r}\right]_{j+l} \\
& =\sum_{l}[U]_{i-p^{k} l}\left[\overparen{S_{\tilde{a}}^{r-k} \delta} R^{\left\langle p^{r-k}\right\rangle}\right]_{j+l} \\
& =\sum_{l}[U]_{i-p^{k} l} \sum_{n}\left(S_{\tilde{a}}^{r-k} \delta\right)_{j+l-p^{r-k} n}[R]_{n} .
\end{aligned}
$$

Let $\lambda=-\left\lfloor p^{-k} \operatorname{deg}(U)\right\rfloor$. Using (4.4), (4.7), (4.5), and (4.8), we obtain

$$
\begin{aligned}
& \left|K_{i} \phi\left(\frac{p^{k} j+i}{p^{r}}\right)-\left(S_{a}^{r} \delta\right)_{p^{k} j+i}\right| \\
& =\left|\sum_{l}[U]_{i-p^{k} l} \sum_{n}[R]_{n}\left(\tilde{\phi}\left(\frac{p^{k} j+i}{p^{r}}-n\right)-\left(S_{\tilde{a}}^{r-k} \delta\right)_{j+l-p^{r-k} n}\right)\right|
\end{aligned}
$$




$$
\begin{aligned}
& \leq \sum_{n}\left|[R]_{n}\right| \sum_{l=\lambda}^{0}\left|[U]_{i-p^{k} l}\right|\left(\left|\tilde{\phi}\left(\frac{p^{k} j+i}{p^{r}}-n\right)-\tilde{\phi}\left(\frac{j+l}{p^{r-k}}-n\right)\right|\right. \\
& \left.+\left|\tilde{\phi}\left(\frac{j+l}{p^{r-k}}-n\right)-\left(S_{\tilde{a}}^{r-k} \delta\right)_{j+l-p^{r-k} n}\right|\right) .
\end{aligned}
$$

We have, for $l \in\{\lambda, \ldots, 0\}$ and $i \in\left\{0,1, \ldots, p^{k}-1\right\}$, that

$$
\left|\frac{p^{k} j+i}{p^{r}}-n-\left(\frac{j+l}{p^{r-k}}-n\right)\right|=\frac{i-p^{k} l}{p^{r}}<\frac{p^{k}+p^{k} \lambda}{p^{r}}=\frac{\lambda+1}{p^{r-k}} \rightarrow 0
$$

independently of $i, j, l$, and $n$ as $r \rightarrow \infty$ and thus, by the uniform continuity of $\tilde{\phi}$, we find that $\left|\tilde{\phi}\left(\frac{p^{k} j+i}{p^{r}}-n\right)-\tilde{\phi}\left(\frac{j+l}{p^{r-k}}-n\right)\right| \rightarrow 0$ independently of $i, j, l$, and $n$ as $r \rightarrow \infty$. By the convergence of subdivision for $\tilde{a}$, we have

$$
\left|\tilde{\phi}\left(\frac{j+l-p^{r-k} n}{p^{r-k}}\right)-\left(S_{\tilde{a}}^{r-k} \delta\right)_{j+l-p^{r-k} n}\right| \rightarrow 0
$$

independently of $j, l$, and $n$ as $r \rightarrow \infty$. Since both $\sum_{n}\left|[R]_{n}\right|$ and $\sum_{l=\lambda}^{0}\left|[U]_{i-p^{k} l}\right|$ are finite constants and not dependent on $r$, we conclude that indeed (1.18) holds with $m=p^{k}$.

We also have, by use of (4.4), Lemma 2.4, as well as the fact that $B(1)=1$, that the SCV satisfies

$$
\begin{aligned}
\sum_{i=0}^{p^{k}-1} K_{i} & =\sum_{i=0}^{p^{k}-1} \sum_{l}\left[W \prod_{j=0}^{k-1} B\left\langle p^{j}\right\rangle\right]_{i-p^{k} l} \\
& =\sum_{j}\left[W \prod_{j=0}^{k-1} B{ }^{\left\langle p^{j}\right\rangle}\right]_{j}=W(1) \prod_{j=0}^{k-1} B^{\left\langle p^{j}\right\rangle}(1)=1,
\end{aligned}
$$

which completes the proof of the theorem.

We can now verify the results conjectured in Example 1.2. We have, in the notation of Theorem 4.3 $p=2$ and $A(z)=\frac{1}{8}\left(1+z^{2}\right)^{2}(3-z), z \in \mathbb{C}$, so that $P(z)=\frac{1}{2}\left(1+z^{2}\right)$, which implies $k=1$, while

and

$$
\begin{aligned}
& B(z)=\frac{1}{4}\left(1+z^{2}\right)(3-z)=\frac{1}{4}\left(3-z+3 z^{2}-z^{3}\right), \quad z \in \mathbb{C}, \\
& \tilde{A}(z)=\frac{1}{8}(1+z)\left(1+z^{2}\right)(3-z)=\frac{1}{8}\left(3+5 z+z^{2}-z^{3}\right), \quad z \in \mathbb{C} .
\end{aligned}
$$

To show subdivision convergence for the mask $\tilde{a}$, we shall again apply Theorem 4.3 and also employ Lemma 3.1. Consider the mask symbol

$$
\tilde{\tilde{A}}(z)=\frac{1}{8}(1+z)^{2}(3-z), \quad z \in \mathbb{C} .
$$

It is known (see e.g. [10, Example 8.5]) that subdivision for the mask $\tilde{\tilde{a}}$ converges. Hence by Theorem 4.3 subdivision for the mask $\tilde{a}$ has 2-subsequence convergence. By noting from Remark 2.5 that $\tilde{W}=E_{0}$, we find

$$
\tilde{W}(z) \tilde{B}(z)=\tilde{B}(z)=\frac{1}{4}\left(3+2 z-z^{2}\right), \quad z \in \mathbb{C} .
$$


Consequently, the SCV is given, according to (4.4), by

and

$$
\begin{aligned}
& \tilde{K}_{0}=\sum_{l}[\tilde{W} \tilde{B}]_{-2 l}=\frac{1}{4}(3-1)=\frac{1}{2} \\
& \tilde{K}_{1}=\sum_{l}[\tilde{W} \tilde{B}]_{1-2 l}=\frac{1}{4}(2)=\frac{1}{2} .
\end{aligned}
$$

Thus $\tilde{K}_{0}=\tilde{K}_{1}$, which, by Lemma 3.1 (d) with $m=n=2$ and $l=1$, implies that subdivision for the mask $\tilde{a}$ has 1-subsequence convergence, i.e. subdivision for $\tilde{a}$ converges.

Returning to the mask $a$, we now have from Theorem 4.3 that 2 -subsequence convergence does indeed occur. Similarly to what was done above, one obtains

and

$$
\begin{aligned}
& K_{0}=\sum_{l}[W B]_{-2 l}=\frac{1}{4}(3+3)=\frac{3}{2} \\
& K_{1}=\sum_{l}[W B]_{1-2 l}=\frac{1}{4}(-1-1)=-\frac{1}{2},
\end{aligned}
$$

as conjectured in Section 1.

Example 4.4. Our next examples illustrates Theorem 4.3 for the case of complexvalued masks. Let $p=2$ and set

$$
A_{\mathbb{C}}(z)=\frac{1}{6}\left(2+(1-2 \boldsymbol{i}) z+\boldsymbol{i} z^{2}+(2+\boldsymbol{i}) z^{3}+z^{4}\right)=\frac{1}{3} P(z)(2+z), \quad z \in \mathbb{C},
$$

where $P(z)=\frac{1}{2}(1-\boldsymbol{i} z)\left(1+\boldsymbol{i} z^{2}\right), z \in \mathbb{C}$, so that, according to Figure 2.1, $P$ is a 2 -GBP factor of level $k=2$. The corresponding reduced mask symbol $\tilde{A}$ is given by

$$
\tilde{A}(z)=\frac{1}{6}(1+z)(2+z)=\frac{1}{6}\left(2+3 z+z^{2}\right), \quad z \in \mathbb{C} .
$$

By Theorem 1.13, the subdivision algorithm $\left(S_{\tilde{a}, p}, \delta\right)$ converges to the corresponding refinable function $\tilde{\phi}$. (This function is well known, being considered in e.g. 9, Chapter 2].) Since $P$ is a 2-GBP factor of level 2, it follows from Theorem 4.3 that subdivision with the mask $a_{\mathbb{C}}$ has 4 -subsequence convergence to the corresponding 2-refinable function $\phi_{\mathbb{C}}$. In the notation of Remark 2.3, we have here $r_{0}=E_{1}$ and $r_{1}(z)=\frac{1-\boldsymbol{i}}{2}(1+\boldsymbol{i} z), z \in \mathbb{C}$, so that

$$
R(z)=\frac{1-\boldsymbol{i}}{4}\left(1+(1+\boldsymbol{i}) z+\boldsymbol{i} z^{2}\right), z \in \mathbb{C} .
$$

Thus we find that $\phi_{\mathbb{C}}$ is given by

$$
\phi_{\mathbb{C}}=(1-i) \tilde{\phi}+2 \tilde{\phi}(\cdot-1)+(1+i) \tilde{\phi}(\cdot-2) .
$$

To calculate the SCV, observe that

$$
B(z)=\frac{1}{3}(2+z) \quad \text { and } \quad W(z)=\frac{E_{3}(z)}{R(z)}=\frac{1+\boldsymbol{i}}{2}(1-\boldsymbol{i} z)
$$

for $z \in \mathbb{C}$. The formula $K_{i}=\sum_{l}\left[W B B^{\langle 2\rangle}\right]_{i-4 l}, i \in \mathbb{Z}_{4}$, obtained from (4.4), now yields the SCV values

$$
K_{0}=\frac{5+3 \boldsymbol{i}}{18}, \quad K_{1}=\frac{3-\boldsymbol{i}}{9}, \quad K_{2}=\frac{2}{9}, \quad \text { and } \quad K_{3}=\frac{3-\boldsymbol{i}}{18} .
$$




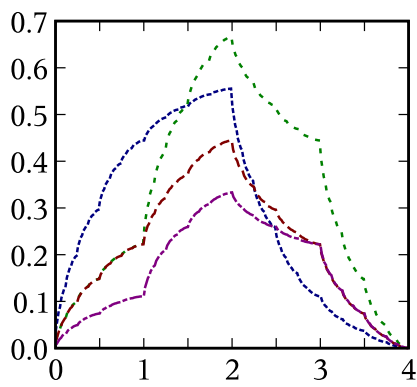

(a)

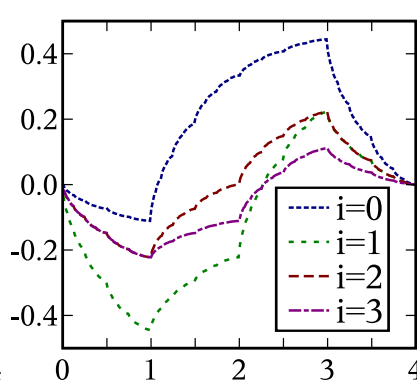

(b)

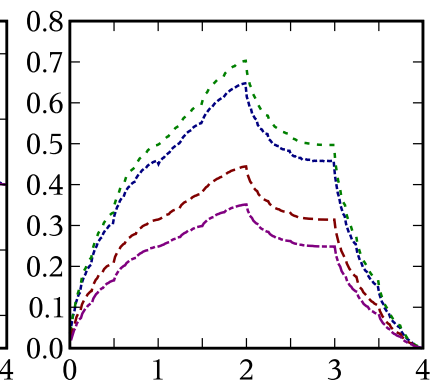

(c)

Figure 4.2. Plots of (a) the real parts, (b) imaginary parts, and (c) magnitudes of $\left(c_{\mathbb{C}}^{(8)}\right)_{4 \cdot+i}, i \in \mathbb{Z}_{4}$, in Example 4.4 .
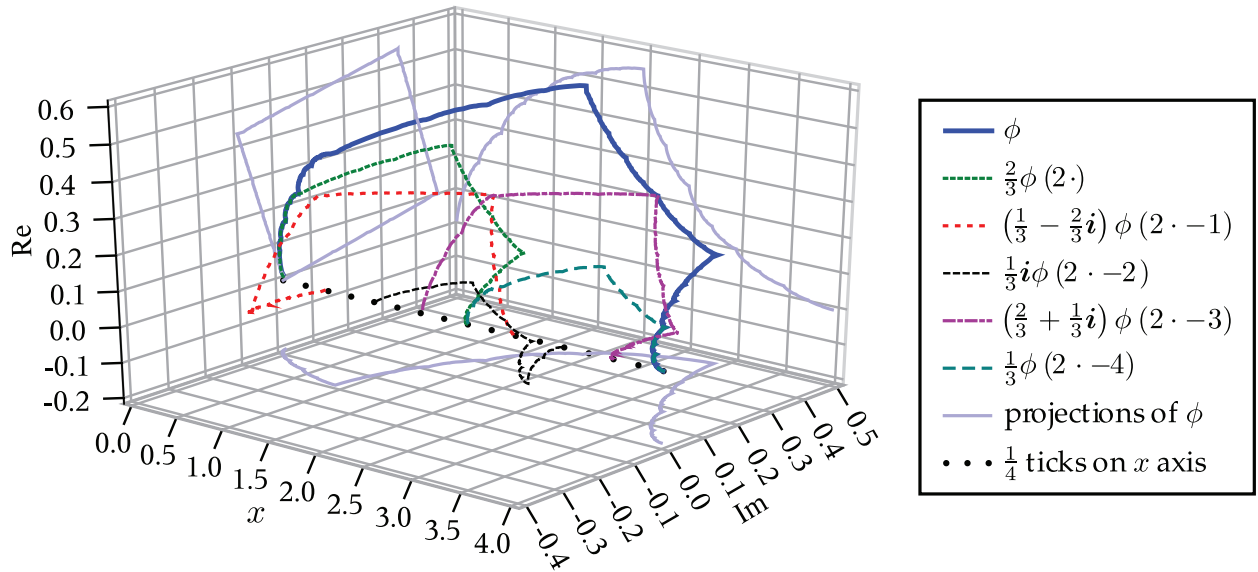

Figure 4.3. A further plot to illustrate the structure of the complex-valued refinable function $\phi_{\mathbb{C}}$ in Example 4.4.

Note in particular that $K_{1}=2 K_{3}$. This result is graphically illustrated in Figure 4.2, where we adopt the notation $c_{\mathbb{C}}^{(r)}=\left(S_{a_{\mathbb{C}}, 2}^{r} \delta\right), r \in \mathbb{Z}_{+}$, while Figure 4.3 illustrates the structure and refinability of the limit function $\phi_{\mathbb{C}}$.

\section{Applications}

It is important to realise that normal subdivision algorithms can easily be adapted to make use of subsequence convergence, as one simply uses normal subdivision and then subsamples the resulting control points to obtain the desired subdivided curve. It must be noted that in practice one must take extra care at the endpoints to account for subsequence convergence.

Example 5.1. We now show another novel (albeit less serious) application of subdivision convergence, namely how it can be used for decorative effects. Fix the dilation factor at 2 and let, for $\beta \in(0,1)$, the mask symbol $A_{\beta}$ be defined by

$$
A_{\beta}(z)=\frac{1}{4}\left(1+z^{2}\right)^{2}(\beta+(1-\beta) z), \quad z \in \mathbb{C},
$$


and take $P=E_{1}^{\langle 2\rangle}$. It can now be verified that the corresponding reduced mask $\tilde{a}_{\beta}$ of Theorem 4.3 is a positive mask, so that by Theorem 1.13, subdivision with the mask $\tilde{a}_{\beta}$ is convergent. Since $P$ is a 2 -GBP factor of level 1 , by Theorem 4.3 it follows that 2 -subsequence convergence will occur for the mask $a_{\beta}$. It can be easily checked that the SCV is given by $K_{0}=\beta$ and $K_{1}=1-\beta$.

In this example, we actually have

$$
c_{2}^{(r)}=\frac{1-\beta}{\beta} c_{2 \cdot+1}^{(r)}=\frac{K_{1}}{K_{0}} c_{2 \cdot+1}^{(r)}, \quad r \in \mathbb{N},
$$

as can be verified by induction. The relationship (5.2) means that if we take a small number of iterations, say 5 or 6 , we obtain a curve which jumps back and forth between the two curves formed by the even and odd indexed entries of the curve. This can give rise to interesting patterns. We illustrate this in Figure 5.1(a), where we subdivide a simple "diamond" shape six times using the mask obtained for $\beta=\frac{3}{4}$.

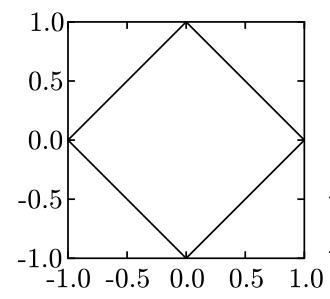

(a)

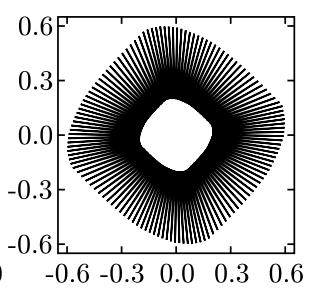

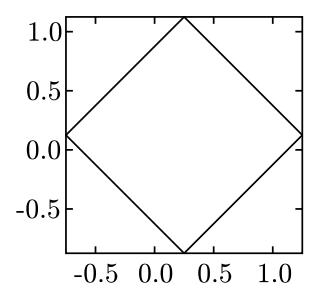

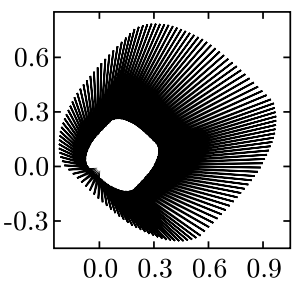

(b)

Figure 5.1. Plots of the initial curve and the sixth iteration of subdivision with $\beta=\frac{3}{4}$ in Example 5.1 for (a) a diamond shape centered on the origin and (b) the diamond shape shifted right and up.

Note that the appearance of the resulting shape is not translation independent, as can be seen from Figure 5.1(b), where we again use $\beta=\frac{3}{4}$ and subdivide 6 times as in Figure 5.1(a), but shift the initial curve a $\frac{1}{4}$ unit right and an $\frac{1}{8}$ unit up. By varying the value of $\beta$, one can also control the ratio between the "even" and "odd" curves according to (5.2).

Of course one can use the same techniques to construct more complex decorations based on $m$-subsequence convergence where $m>2$.

An issue that might be of interest for future research is the consideration of subsequence convergence in the matrix subdivision case as considered in [7] and the other references listed in the introduction.

\section{NeSted SETS Of REFinement MASKS}

The results of this chapter allow us to build a nested sequence of sets of refinement masks. Let $R M(p)$ denote the set of all $p$-refinement mask symbols corresponding to continuous $p$-refinable functions. Let $R M S(p, k)$ denote the set of mask symbols for which the corresponding $p$-subdivision algorithm has $p^{k}$-subsequence convergence. Specifically, $R M S(p, 0)$ denotes the set of mask symbols for which $p$-subdivision converges. 
It is well known (see e.g. 10]) that $R M S(2,0) \varsubsetneqq R M(2)$. The following results give further insight into the nature of the set $R M(p) \backslash R M S(p, 0)$.

In view of Theorem 3.3, we know that $R M S(p, k) \subset R M(p)$ for any $k \in \mathbb{Z}_{+}$. Furthermore, from Lemma 3.1(c) it follows that $R M S(p, k-1) \subset R M S(p, k)$ for $k \in \mathbb{N}$. Since $\left(E_{p-1}\right)^{m} \in R M S(p, 0)$ for $p \in \mathbb{N}, p \geq 2$, and $m \in \mathbb{N}$, we thus obtain, for $p \in \mathbb{N}, p \geq 2$,

$$
\emptyset \neq R M S(p, 0) \subset R M S(p, 1) \subset \cdots \subset R M S(p, k) \subset \cdots \subset R M(p) .
$$

The next result shows that $\left\{R M S(p, k): k \in \mathbb{Z}_{+}\right\}$is a properly nested sequence.

Theorem 6.1. For $k \in \mathbb{N}$, the relation $R M S(p, k-1) \varsubsetneqq R M S(p, k)$ holds.

Proof. Let the dilation factor $p \in \mathbb{Z}, p \geq 2$, be given and let $B(z)=\frac{2}{3}+\frac{1}{3} z, z \in \mathbb{C}$. Define, for $k \in \mathbb{Z}_{+}, A_{k}=E_{p-1}^{\left\langle p^{k}\right\rangle} B$. Then $A_{0}$ is the symbol of a positive mask for which the sum rules hold, so that Theorem 1.13 guarantees the convergence of the corresponding subdivision algorithm. Then for any $k \in \mathbb{Z}_{+}$, since $E_{p-1}^{\left\langle p^{k}\right\rangle}$ is a $p$-GBP factor of level $k$ and the reduced mask $\tilde{A}_{k}$ is exactly $A_{0}$, we know from Theorem 4.3 that the subdivision algorithm for $A_{k}$ has $p^{k}$-subsequence convergence to the associated refinable function $\phi_{k}$, so that $A_{k} \in R M S(p, k)$. To show that $p^{k-1}$ subsequence convergence does not occur for $A_{k}$ when $k \geq 1$, we first show that the SCV satisfies $K_{0} \neq K_{p^{k-1}}$. To do so, define

$$
M_{k}=\prod_{j=0}^{k-1} B^{\left\langle p^{j}\right\rangle}, \quad k \in \mathbb{N} .
$$

Then for $k \in \mathbb{Z}, k \geq 2$, it follows that $M_{k}=B M_{k-1}^{\langle p\rangle}$, from which we deduce by (1.8c) and the definition of $B$ that

$$
\left[M_{k}\right]_{p j}=\sum_{l}\left[M_{k-1}\right]_{l}[B]_{p j-p l}=\frac{2}{3}\left[M_{k-1}\right]_{j}, \quad k \in \mathbb{Z}, k \geq 2 .
$$

Since $[B]_{p j}=2[B]_{p j+1}$ and $B=M_{1}$, we obtain, by repeated application of (6.2), for $k \in \mathbb{Z}, k \geq 2$,

$$
\left[M_{k}\right]_{p^{k} j}=\left(\frac{2}{3}\right)^{k-1}\left[M_{1}\right]_{p j}=2\left(\frac{2}{3}\right)^{k-1}\left[M_{1}\right]_{p j+1}=2\left[M_{k}\right]_{p^{k} j+p^{k-1}}, \quad j \in \mathbb{Z},
$$

allowing us, in view also of Remark 2.5. to deduce that

$$
K_{0}=\sum_{j}\left[M_{k}\right]_{-p^{k} j}=2 \sum_{j}\left[M_{k}\right]_{p^{k-1}-p^{k} j}=2 K_{p^{k-1}}
$$

for any given $k \in \mathbb{N}$. Since it can also be verified that $K_{0} \neq 0$, it follows that $K_{0} \neq$ $K_{p^{k-1}}$, so that by Lemma 3.1(d) we conclude that the subdivision algorithm for $A_{k}$ does not have $p^{k-1}$-subsequence convergence to $p^{-1} \phi_{k}$. Now suppose that $A_{k} \in$ $R M S(p, k-1)$. Then there is a $p$-refinable function $\psi_{k}$ such that the subdivision algorithm for $A_{k}$ has $p^{k-1}$-subsequence convergence to $\psi_{k}$. Then by Lemma 3.1(c), subdivision for $A_{k}$ has $p^{k}$-subsequence convergence to $p \psi_{k}$, which by Lemma 3.1.(b) means that $\psi_{k}=p^{-1} \phi_{k}$, contradicting the fact that subdivision for $A_{k}$ does not have $p^{k-1}$-subsequence convergence to $p^{-1} \phi_{k}$. Thus $A_{k} \notin R M S(p, k-1)$, showing that $A_{k} \in R M S(p, k) \backslash R M S(p, k-1)$. With (6.1), this gives the desired result. 
Using subsequence convergence in subdivision allows us to plot all the $p$-refinable functions corresponding to masks in $R M S(p, \infty):=\bigcup_{k \in \mathbb{Z}_{+}} R M S(p, k)$, which, by Theorem 6.1 and the uniqueness results Theorem 1.9(a) and Lemma 1.10, is a proper superset of the $p$-refinable functions that can be plotted by normal subdivision. An interesting remaining open problem is to determine the nature of the set $R M(p) \backslash R M S(p, \infty)$.

\section{REFERENCES}

1. Lothar Berg and Gerlind Plonka, Compactly supported solutions of two-scale difference equations, Lin. Alg. Appl. 275 (1998), 49-75. MR.1628382 (99h:39002)

2. $ـ$ Some notes on two-scale difference equations, Functional Equations \& Inequalities (T.M. Rassias, ed.), Kluwer Academic Publishers, 2000. MR.1792070 (2002f:39049)

3. Alfred S. Cavaretta, Wolfgang Dahmen, and Charles A. Micchelli, Stationary subdivision, Mem. Amer. Math. Soc. 93 (1991), no. 453. MR1079033 (92h:65017)

4. W. Dahmen and C. A. Micchelli, Biorthogonal wavelet expansions, Constr. Approx. 13 (1997), 293-328. MR1451708 (99c:39039)

5. Ingrid Daubechies and Jeffrey C. Lagarias, Two-scale difference equations I. Existence and global regularity of solutions, SIAM J. Math. Anal. 22 (1991), no. 5, 1388-1410. MR1112515 (92d:39001)

6. W.d.V. de Wet, On the analysis of refinable functions with respect to mask factorisation, regularity and corresponding subdivision convergence., Ph.D. thesis, University of Stellenbosch, 2007.

7. Vera Latour, Jürgen Müller, and Werner Nickel, Stationary subdivision for general scaling matrices, Math. Z. 227 (1998), 645-661. MR1621951 (99c:65027)

8. Wayne Lawton, S. L. Lee, and Zuowei Shen, Characterization of compactly supported refinable splines, Adv. Comp. Math. 3 (1995), 137-145. MR.1314906 (95m:41020)

9. Charles A Micchelli, Mathematical aspects of geometric modeling, SIAM, 1995. MR1308048 (95i:65036)

10. Marian Neamtu, Convergence of subdivision versus solvability of refinement equations, East J. Approx. 5 (1999), no. 2, 183-210. MR1705396 (2000j:42054)

11. Thomas Sauer, Differentiability of multivariate refinable functions and factorization, Adv. Comput. Math. 25 (2006), 211-235. MR2231702(2007e:42049)

Department of Mathematical Sciences, Mathematics Division, Private Bag X1, MatieLAND 7602, South Africa 